\title{
A Multicenter Survey on the Trend of Chest CT Scan Utilization: Tracing the First Footsteps of COVID-19 in Iran
}

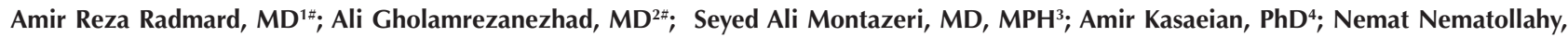
MD ${ }^{5}$; Roghieh Molaee Langrudi, MD $^{6}$; Reza Javad Rashid, MD ; Alireza Dehghan, MD ${ }^{8}$; Ali Hekmatnia, MD ${ }^{9}$; Ali Shakourirad, MD ${ }^{10}$; Masoud Pezeshki Rad, MD ${ }^{11}$; Reza Nafisi Moghadam, MD²; Hashem Sharifian, MD ${ }^{13}$; Ahmad Enhesari, MD ${ }^{14}$; Marzieh Aalinezhad, MD ${ }^{9}$; Ghazaleh Jamalipour Soufi, MD ${ }^{9}$; Alireza Shakibafard, MD ${ }^{15}$; Maryam Mohammadzadeh, MD ${ }^{13}$; Reza Jalli, MD ${ }^{8}$; Mehrdad Bakhshayeshkaram, MD ${ }^{16}$; Taraneh Faghihi Langroudi, MD"7; Masoomeh Raoufi, MD ${ }^{18}$; Alireza Abrishami, MD ${ }^{19}$; Pooneh Dehghan, MD ${ }^{20}$; Hooman Bahrami-Motlagh, $\mathrm{MD}^{21}$; Hassan Hashemi, $\mathrm{MD}^{22}$; Morteza Sanei Taheri, $\mathrm{MD}^{23^{+}}$

\author{
'Department of Radiology, Shariati Hospital, Tehran University of Medical Sciences, Tehran, Iran \\ ${ }^{2}$ Keck School of Medicine, University of Southern California, Los Angeles, CA, USA \\ ${ }^{3}$ Department of Radiology, Mayo Clinic, Florida, USA \\ ${ }^{4}$ Department of Biostatistics and Epidemiology, Hematology-Oncology and Stem Cell Transplantation Research Center, Shariati Hospital, \\ Tehran University of Medical Sciences, Tehran, Iran \\ ${ }^{5}$ Department of Radiology, 5 Azar Hospital, Golestan University of Medical Sciences, Gorgan, Iran \\ ${ }^{6}$ Diagnostic Radiology Department, Poursina Hospital, Guilan University of Medical Sciences, Rasht, Iran \\ ${ }^{7}$ Department of Radiology, Imam Reza hospital, Tabriz University of Medical Sciences, Tabriz, Iran \\ ${ }^{8}$ Department of Radiology, Namazi Hospital, Shiraz University of Medical Sciences, Shiraz, Iran \\ ${ }^{9}$ Radiology Department, School of Medicine, Isfahan University of Medical Sciences, Isfahan, Iran \\ ${ }^{10}$ Department of Radiology, Sina Hospital, Tehran University of Medical Sciences, Tehran, Iran \\ "Department of Radiology, Imam Reza Hospital, Mashhad University of Medical Sciences, Mashhad, Iran \\ ${ }^{12}$ Department of Radiology, Shahid Sadoughi Hospital, Shahid Sadoughi University of Medical Sciences, Yazd, Iran \\ ${ }^{13}$ Department of Radiology, Amir A'lam Hospital, Tehran University of Medical Sciences, Tehran, Iran \\ ${ }^{14}$ Clinical Research Unit, Afzalipour Hospital, Kerman University of Medical Sciences, Kerman, Iran \\ ${ }^{15}$ TABA Medical Imaging Center, Shiraz, Iran \\ ${ }^{16}$ Chronic Respiratory Diseases Research Center, National Research Institute of Tuberculosis and Lung Diseases (NRITLD), Shahid Beheshti \\ University of Medical Sciences, Tehran, Iran \\ ${ }^{17}$ Department of Radiology, Shahid Modarres Hospital, Shahid Beheshti University of Medical Sciences, Tehran, Iran \\ ${ }^{18}$ Department of Radiology, Imam Hossein Hospital, Shahid Beheshti University of Medical Sciences, Tehran, Iran \\ ${ }^{19}$ Department of Radiology, Shahid Labbafinejad Hospital, Shahid Beheshti University of Medical Sciences, Tehran, Iran \\ ${ }^{20}$ Department of Radiology, Taleghani Hospital, Shahid Beheshti University of Medical Sciences, Tehran, Iran \\ ${ }^{21}$ Department of Radiology, Loghman Hakim Hospital, Shahid Beheshti University of Medical Sciences, Tehran, Iran \\ ${ }^{22}$ Advanced Diagnostic and Interventional Radiology Research Center (ADIR), Tehran University of Medical Sciences, Tehran, Iran \\ ${ }^{23}$ Department of Radiology, Shohada-E-Tajrish Hospital, Shahid Beheshti University of Medical Sciences, Tehran, Iran
}

\begin{abstract}
Background: Chest computed tomography (CT) scan has been used widely to diagnose COVID-19 in Iran

Objectives: To trace the footsteps of COVID-19 in Iran by exploring the trend in using chest CT scans and its economic impact on radiology departments.

Methods: In this cross-sectional study, the number of imaging examinations from 33 tertiary radiology departments in 9 large cities of Iran was collected from September 23, 2019 to March 20, 2020 (Months 1 to 6) and the corresponding months in 2018-2019.

Results: A 50.2\% increase was noted in the chest CT scan utilization in 2019-2020 compared to 2018-2019. This increase was $+15 \%$, $+15 \%,+27 \%,+2 \%,+1 \%$ in Months $1-5$ of 2019-2020, respectively. In Month 6 of 2019-2020, a 251\% increase in the acquisition of chest CT scans was observed compared to the Month 6 of 2018-2019. Following negative balance of revenue from Month 1 to 5 with respect to the inflation rate, the total income in Month 6 was further $1.5 \%$ less than the same Month in 2018-19.

Conclusion: The observed peak in chest CT utilization in Month 3 prior to the surge in Month 6 could be explained by the seasonal influenza. However, unawareness about an emerging viral disease, i.e. COVID-19, might have underutilized chest CT in Months 4 and 5 before the official announcement in Month 6 . The unbalanced increase in the workload of radiology departments in the shortage of cardiothoracic radiologists with the simultaneous decrease in income initiated a vicious cycle that worsened the economic repercussions of the pandemic.

Keywords: COVID-19, Hospital, Multicenter Study, Multidetector computed tomography, Radiology department, Public health Cite this article as: Radmard AR, Gholamrezanezhad A, Montazeri SA, Kasaeian A, Nematollahy N, Molaee Langrudi R, et al. A multicenter survey on the trend of chest CT scan utilization: tracing the first footsteps of COVID-19 in Iran. Arch Iran Med. 2020;23(11):787-793. doi: 10.34172/aim.2020.105
\end{abstract}

Received: July 18, 2020, Accepted: September 6, 2020, ePublished: November 1, 2020 


\section{Introduction}

The coronavirus disease 2019 (COVID-19) pandemic has caused 593000 deaths in 13.9 million infected individuals as of July 17, 2020, ${ }^{1}$ which has resulted in an unprecedented increasing workload for the healthcare system. The need for accurate and rapid tests to diagnose the severe acute respiratory syndrome coronavirus 2 (SARS-CoV-2) is crucial for optimal clinical management of the patients and effective control of the COVID-19 pandemic.

Iran was one of the first countries struggling with surging numbers of COVID-19 patients. ${ }^{2}$ At the time of writing, more than 269000 individuals have been diagnosed with the disease and 13000 have died in Iran. ${ }^{1}$ Although these numbers appear to be significantly underestimated, ${ }^{3}$ Iran suffers from the highest burden of the disease in the Middle East.

Limited availability of the reverse-transcriptase polymerase-chain-reaction (RT-PCR) test kits for SARSCoV-2 and the reportedly higher sensitivity of chest computed tomography $(\mathrm{CT})^{4}$ in patients with a high pretest probability of the disease ${ }^{5}$ forced the healthcare system to implement chest CT in the initial diagnostic workup of patients with suspect COVID-19. Therefore, tracing the number of chest CT studies since early fall 2019 could potentially give us the footsteps of the disease.

The implementation of chest CT in the initial workup of patients with respiratory symptoms has been a valuable step in controlling the pandemic. A notable shift to the imaging of chest with a possible drop in imaging of other organs, however, may raise concerns on the job security for the radiologists who have been involved in other subspecialties without enough experience in thoracic imaging. The economic consequences could also be a matter of concern for the radiology departments, which have already been influenced by economic sanctions and currency depreciations in Iran.

This study aimed to explore the trend and pattern of utilization of imaging modalities in 2019-2020 in 9 big cities of Iran with a total population of more than 20 million. The trend in chest CT utilization is applied to trace COVID-19 footstep and address the pros and cons of radiology subspecialization. The economic impact of the change in workflow and imaging requisitions on the radiology departments and imaging centers is also studied.

\section{Materials and Methods}

Data Source

For this cross-sectional study, data were obtained from health information systems and picture archiving and communication systems at 33 radiology departments/ imaging centers in 9 large cities in Iran, as capitals of the provinces. These centers were tertiary main referral imaging centers in their corresponding provinces including radiology departments of 28 university teaching hospitals and three major private imaging centers (Table 1). The names and populations of these cities are as follows: Tehran (8737510), Mashhad (3,372660), Isfahan (2 243249), Shiraz (1 869001), Tabriz (1733033), Rasht (956971), Kerman (738724), Yazd (656474), and Gorgan (480541). With a total population of 20788163 , these cities constitute 5 out of the 6 most populated cities and more than $26 \%$ and $35 \%$ of total and urban population in Iran, respectively. ${ }^{6}$

\section{Patient and Public Involvement}

The public were not involved in the design, or conduct, or recruitment the data for this study. The results of this study will be disseminated to the public according to the journal's policies.

\section{Data Classification}

The number of obtained imaging modalities was collected on a monthly basis per two 6-month periods, which correspond to the flu season. The first period, i.e. 2018-2019, started from September 2018 to March 2019 and the second, i.e. 2019-2020, covers September 2019 to March 2020. Due to the difference in the Persian (Solar Hijri) and Gregorian calendars, the data from each Month are depicted in the numerical order. For example, the month of 'Mehr' in Persian Calendar year 1397 corresponds to September 23 to October 22, 2018 in Gregorian Calendar, which was considered as "Month 1 " in this study. Accordingly, Month $2($ Aban $)=$ October 23 to November 21; Month 3 (Azar) = November 22 to December 21; Month 4 (Dey) = December 22 to January 20; Month 5 (Bahman) = January 21 to February 19; and Month 6 (Esfand) = February 19 to March 20. The same order applies to the second 6-month period (2019-2020) from September 23, 2019 to March 20, 2020.

The data were categorized as: 1 . Chest CT scan (CT with or without IV contrast, high-resolution CT, CT angiography of the pulmonary arteries, etc.); 2. Brain CT scan [with or without IV contrast, CT angiography (CTA), CT venography (CTV), etc.]; 3. Abdominopelvic CT scan (with or without IV contrast, CTA, CTV, CT Urography, etc.); and 4. Others (facial, orbital, paranasal sinuses, temporal bone CT, spine, and extremities). The total number of magnetic resonance imaging (MRI) studies, regardless of the body part, was also collected.

\section{Data Analysis}

STATA statistical software, Release 14, StataCorp LLC, was used. The trend in utilization of each imaging modality was depicted. The income of radiology departments was determined as follows. The cost per each modality was calculated by the total number of the imaging studies multiplied by the official tariffs set by the Iranian health authorities in 2018-2019 and 2019-2020. Then, the costs of all imaging studies were summed up per Month in 2019-2020 and compared to that in 2018-2019. A Heat 
map figure was used to illustrate the ratio of two odds according to the following formula:

Chest odds ratio $=\frac{\text { odds of ordered chest } C T \text { to the ordered } C T \text { scans for all other organs in month } 6 \text { of } 2019-2020}{\text { odds of ordered }}$

Table 1. Characteristics of the Enrolled Hospitals and Imaging Centers That Provided Data for the Study

\begin{tabular}{|c|c|c|c|c|c|c|c|c|}
\hline Center/Hospital & City, Province & Center/Hospital Type & $\begin{array}{c}\text { General } \\
\text { Beds/ICU } \\
\text { Beds } \\
\end{array}$ & $\begin{array}{c}\text { CT } \\
\text { Scanners } \\
\text { No. } \\
\end{array}$ & $\begin{array}{c}\text { MRI } \\
\text { Machines } \\
\text { No. } \\
\end{array}$ & $\begin{array}{c}\text { General } \\
\text { Radiologists }\end{array}$ & $\begin{array}{l}\text { Fellowship- } \\
\text { Trained } \\
\text { Radiologist } \\
\end{array}$ & $\begin{array}{c}\text { In Training } \\
\text { Residents }\end{array}$ \\
\hline Shariati Hospital & Tehran, Tehran & Teaching & $520 / 73$ & 2 & 1 & 7 & 2 & 28 \\
\hline Sina Hospital & Tehran, Tehran & Teaching & $486 / 51$ & 2 & 1 & 7 & 1 & 27 \\
\hline Amir A'lam Hospital & Tehran, Tehran & Teaching & $280 / 28$ & 1 & 1 & 3 & 1 & 20 \\
\hline Shohada-E-Tajrish Hospital & Tehran, Tehran & Teaching & $485 / 39$ & 2 & 1 & 7 & 2 & 25 \\
\hline Masih Daneshvari Hospital & Tehran, Tehran & Teaching & $330 / 33$ & 1 & 0 & 6 & 0 & 4 \\
\hline Shahid Modarres Hospital & Tehran, Tehran & Teaching & $265 / 38$ & 1 & 0 & 3 & 2 & 5 \\
\hline Shahid Labbafinejad Hospital & Tehran, Tehran & Teaching & $225 / 10$ & 1 & 0 & 3 & 0 & 5 \\
\hline Taleghani Hospital & Tehran, Tehran & Teaching & $481 / 20$ & 1 & 0 & 3 & 0 & 10 \\
\hline Loghman Hakim Hospital & Tehran, Tehran & Teaching & $365 / 32$ & 1 & 0 & 4 & 0 & 15 \\
\hline Imam Hossein Hospital & Tehran, Tehran & Teaching & $605 / 22$ & 1 & 1 & 4 & 0 & 25 \\
\hline Mofid Hospital & Tehran, Tehran & Teaching & $219 / 12$ & 1 & 0 & 1 & 0 & 5 \\
\hline Mahdieh Hospital & Tehran, Tehran & Teaching & $134 / 7$ & 1 & 0 & 2 & 0 & 6 \\
\hline Akhtar Hospital & Tehran, Tehran & Non-teaching (Public) & $114 / 8$ & 1 & 0 & 1 & 0 & 0 \\
\hline Torfeh Hospital & Tehran, Tehran & Non-teaching (Public) & $110 / 0$ & 1 & 0 & 1 & 0 & 0 \\
\hline Mofatteh Hospital & Tehran, Tehran & Non-teaching (Public) & $148 / 7$ & 1 & 1 & 1 & 0 & 0 \\
\hline Pakdasht Hospital & Tehran, Tehran & Non-teaching (Public) & $157 / 7$ & 1 & 0 & 1 & 0 & 0 \\
\hline Damavand Hospital & Tehran, Tehran & Non-teaching (Public) & $108 / 0$ & 1 & 0 & 1 & 0 & 0 \\
\hline Shohada Gomnam Hospital & Tehran, Tehran & Non-teaching (Public) & $120 / 0$ & 1 & 0 & 1 & 0 & 0 \\
\hline Imam Reza Hospital & $\begin{array}{l}\text { Mashhad, Khorasan- } \\
\text { e-Razavi }\end{array}$ & Teaching & $1028 / 97$ & 1 & 1 & 6 & 2 & 44 \\
\hline Amin Hospital & Isfahan, Isfahan & Teaching & $160 / 39$ & 1 & 0 & 3 & 0 & 80 \\
\hline Ayatollah Kashani Hospital & Isfahan, Isfahan & Teaching & $220 / 30$ & 2 & 1 & 3 & 1 & 80 \\
\hline Sepahan Imaging Center & Isfahan, Isfahan & Private & $\mathrm{N} / \mathrm{A}$ & 1 & 2 & 3 & 1 & $\mathrm{~N} / \mathrm{A}$ \\
\hline Namazi Hospital & Shiraz, Fars & Teaching & $824 / 91$ & 3 & 2 & 11 & 5 & 87 \\
\hline TABA Imaging Center & Shiraz, Fars & Private & $\mathrm{N} / \mathrm{A}$ & 1 & 1 & 8 & 1 & $\mathrm{~N} / \mathrm{A}$ \\
\hline Imam Reza Hospital & $\begin{array}{l}\text { Tabriz, Azarbayjan- } \\
\text { e-Sharghi }\end{array}$ & Teaching & $800 / 129$ & 3 & 1 & 11 & 2 & 60 \\
\hline Razi Hospital & Rasht, Guilan & Teaching & $300 / 10$ & 1 & 0 & 3 & 0 & 10 \\
\hline Poursina Hospital & Rasht, Guilan & Teaching & $263 / 29$ & 2 & 1 & 3 & 0 & 9 \\
\hline Afzalipour Hospital & Kerman, Kerman & Teaching & $505 / 120$ & 1 & 0 & 6 & 0 & 16 \\
\hline Sadoughi Hospital & Yazd, Yazd & Teaching & $520 / 48$ & 1 & 1 & 4 & 2 & 32 \\
\hline Rahnemun Hospital & Yazd, Yazd & Teaching & $220 / 36$ & 1 & 1 & 2 & 0 & 6 \\
\hline 5 Azar Hospital & Gorgan, Golestan & Teaching & $400 / 30$ & 2 & 0 & 6 & 0 & 13 \\
\hline Golestan Imaging Center & Gorgan, Golestan & Private & $\mathrm{N} / \mathrm{A}$ & 1 & 1 & 3 & 0 & 9 \\
\hline Sayyad Shirazi Hospital & Gorgan, Golestan & Teaching & $340 / 35$ & 1 & 0 & 4 & 2 & 13 \\
\hline
\end{tabular}

\section{Results}

Total Number of Imaging Modalities in 2019-2020 and 2018-2019

The total number of CT and MRI studies in the second period decreased by $0.3 \%$ compared to the previous period: 430617 and 431817 in 2019-2020 and 2018-2019, respectively. Abdominopelvic CT, brain CT, and MRI scans decreased by $4.3 \%, 10.8 \%$, and $13.9 \%$, in 2019 2020 compared to 2018-2019, respectively. In contrast, a more than $50.2 \%$ increase was seen in the total number of chest CT scans in 2019-2020 compared to 2018-2019 (Figure 1).

Trend in the Number of CT scans

The total number (percentage) of chest CT increased by $1274(+14.6 \%), 1243(+15.1 \%), 2443(+27.3 \%)$, $191(+1.9 \%), 62(+0.7 \%)$ and $21848(+251.5 \%)$ in the $1^{\text {st }}$ to $6^{\text {th }}$ Months, respectively, in the $2019-2020$ period, 


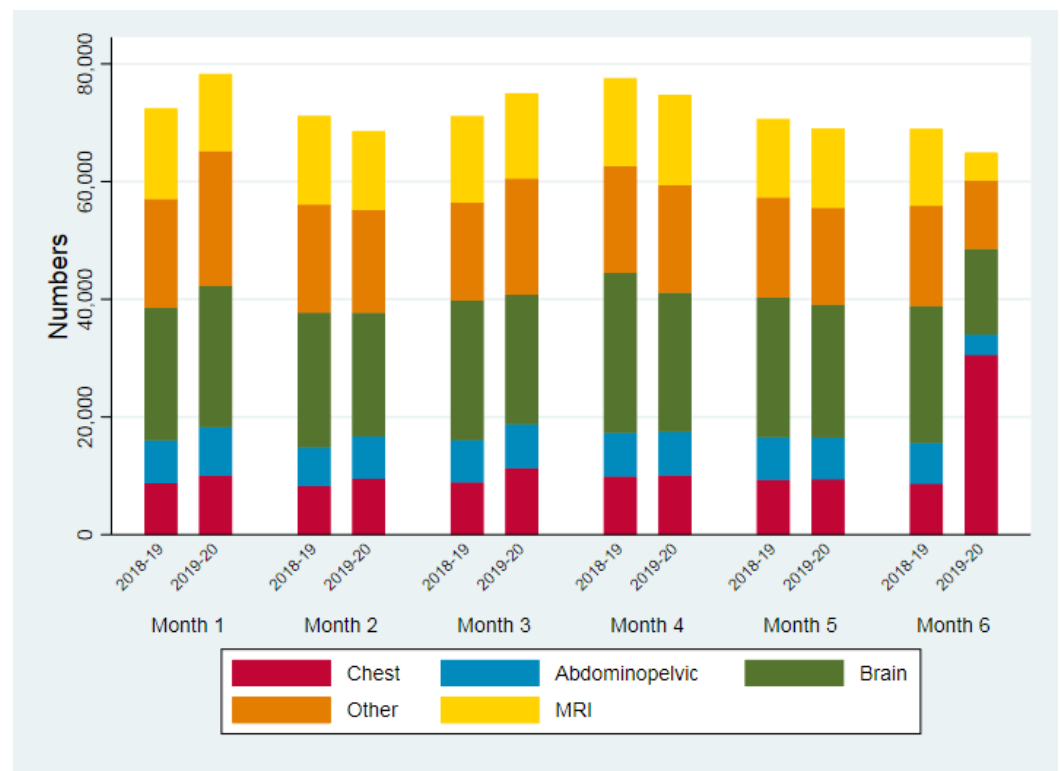

Figure 1. Stacked Bar Chart to Show the Number of CT Studies Per Organ and the Total Number of MRI Examinations from Month 1 To 6 in the Year $2018-$ 2019 Compared to the Year 2019-2020.

compared to the chest $\mathrm{CT}$ scans in the corresponding Months in 2018-2019 (Figure 2). The lowest chest odds ratios were 2.15 and 2.64, which corresponded to Fars and East Azerbaijan provinces, respectively. The highest chest odds ratios were 18.83 and 18.74 , which were seen in Gorgan (Golestan province) and Rasht (Guilan province), respectively (Figure 3). In 2018-2019, brain CT was the most common CT imaging study used, followed by others, chest, and abdominopelvic exams. This pattern was unchanged until Month 6, when a substantial increase was noted in the percentage of chest CT scans.

The number of abdominopelvic CT scans was higher in Months 1, 2, and 3 in 2019-2020 than in the same Months of 2018-2019. A substantial decrease in the number of abdominopelvic CT scans was seen in Month 6 in 2019-2020 compared to the same Month in 2018-

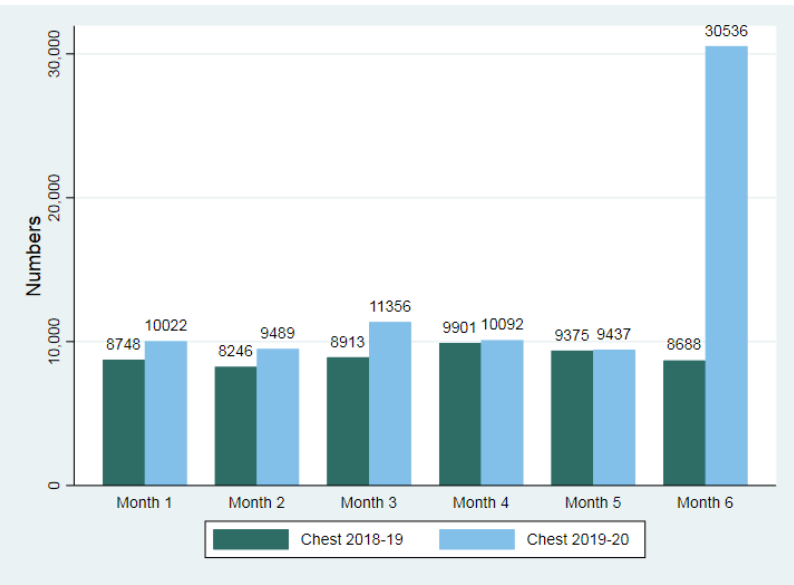

Figure 2. Bar Chart to Illustrate the Total Number of Chest CT Studies in All Departments from Month 1 to 6 in the Year 2018-2019 Compared to the Year 2019-2020.
2019. Similar patterns were seen in brain and other CT scans in 2019-2020 compared to 2018-2019 (Figure 4).

Trend in the Number of MRI Examinations

The total number of MRI examinations was 4712 in Month 6 of 2019-2020 period, which showed a 63.9\% decrease compared to the same Month of the previous year.

Analysis of the Income

The total income of all radiology departments and imaging centers was IRR 32.1 and IRR 31.6 billion in 2018-2019

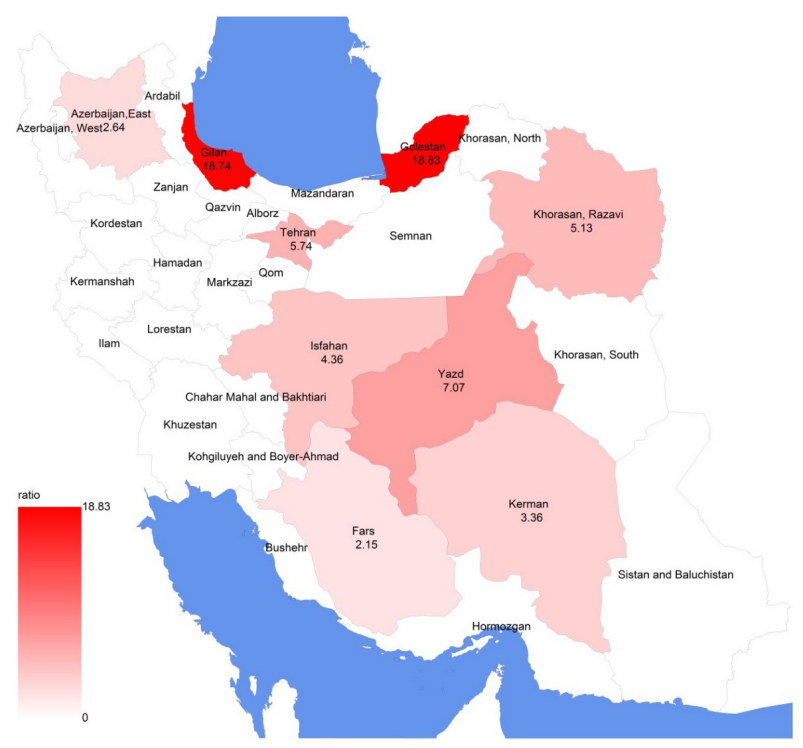

Figure 3. Heat Map to Illustrate Ratios of Two Odds Including Odds of Chest CT Numbers to the Number of Non-Chest CT Studies in Month 6, 2019-2020 Divided by the Same Odds in Month 6 of 2018-19 for Each of 9 Provinces. 


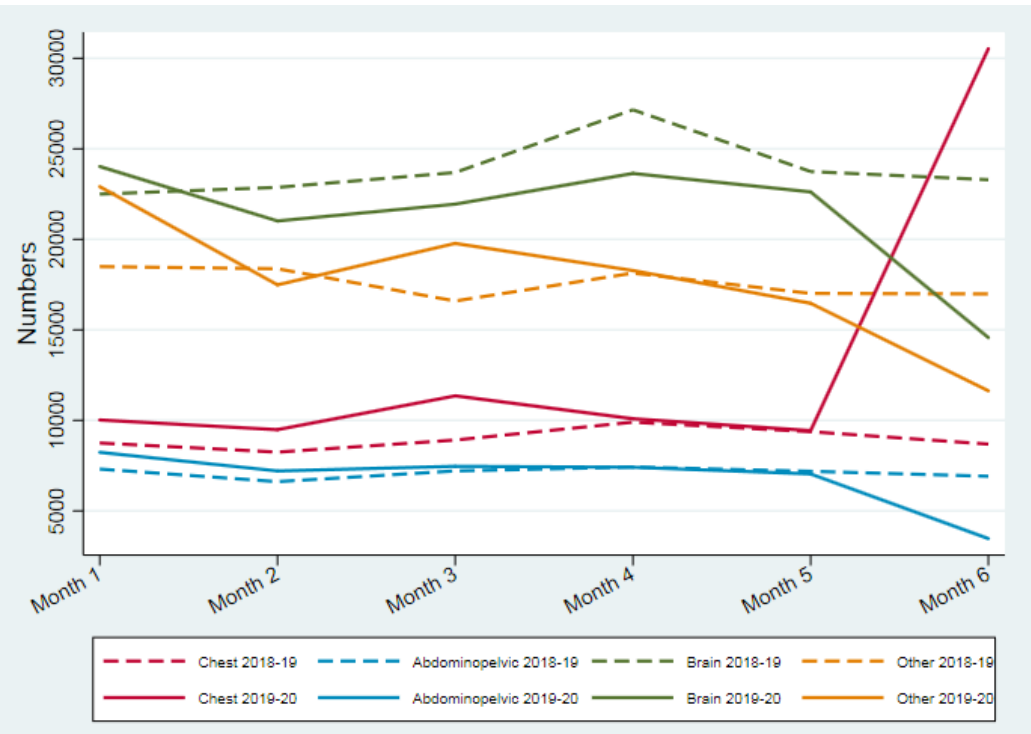

Figure 4. Line Chart to Display Trends in the Number of CT Studies Per Organ in All Departments from Month 1 to 6 in the Year 2018-2019 Compared to the Year 2019-2020.

and 2019-2020, respectively. This income was increased in Months 1 to 5 of 2019-2020 compared to the same period in 2018-2019. The last Month, however, showed a decrease in the income by $1.5 \%$ (Figure 5 ).

\section{Discussion}

Catching the First Glimpse of Pandemic

The outbreak of COVID-19 in China was first reported in late December 2019.7 Some reporters, however, recently figured out that the patient zero might be traced back to November 17, 2019. ${ }^{8}$ Although the first case of COVID-19 in Iran was confirmed on February 19, 2020, ${ }^{2}$ tracing the actual first patient has been cumbersome because it was an unknown respiratory disease outbreak during the flu season. One way to trace the first signs of the outbreak is to look for the number of diagnostic tests ordered in the referral hospitals. It has been very difficult to monitor the disease outbreak in the large cities of Iran, due to the limited number of available molecular diagnostic assays and RT-PCR kits. Therefore, a chest CT scan was initially implemented in the diagnostic workup of the patients with respiratory signs and symptoms in Iran., 4

In this survey, the total number of chest $\mathrm{CT}$ acquisitions in the 2019-2020 period was 50\% higher than the same period of 2018-2019. This increase consisted of $15 \%-27 \%$ rise in the first three Months of 2019-2020 compared to the previous period, followed by a twomonth trend of unchanged volume. A substantial 251\% increase in the number of CT scans was seen in Month 6 of 2019-2020 compared to Month 6 of 2018-2019. This pattern could be explained by two hypotheses: first, higher incidence of seasonal influenza-associated pneumonia with the highest peak in Month 3. Seasonal waves of influenza are commonly expected every year, which may present a complex spatiotemporal pattern depending on the biological, sociodemographic, and environmental factors. ${ }^{9}$ The second hypothesis based on the higher peak of chest CT utilization in Month 3 might be attributed to the other causes of acute respiratory diseases like unknown viruses (e.g. COVID-19). However, unawareness of the healthcare system, especially physicians, about the presence of a confirmed new virus in Iran might have led to a relative decrease in chest CT utilization in Months 4 and 5 before a considerable surge in Month 6. On the other hand, a part of notable overutilization of chest CT in Iran in 6 was derived from the panic of being infected by COVID-19, which resulted in significantly increased requisitions without sufficient clinical indications. Nevertheless, the hypothesis of earlier COVID-19 spread to Iran could have been supported if the earlier peak in Month 3 had been continuously extended to Months 4 and 5 , then to the highest peak in Month 6 .

The Pattern of Utilization of Imaging Studies and Economic and Workload Consequences

In contrast to chest CT scans, radiology departments have faced a remarkable reduction in the number of brain CT, abdominopelvic CT, other CT scans, and MRI examinations in 2019-2020 compared to 2018-2019. In a large healthcare system in New York, the United States, a substantial decline, i.e. $28 \%$, was reported in all imaging volumes. Mammography, MRI, nuclear medicine, and ultrasound witnessed the largest decrease in acquisition. However, more detailed information was not provided about the change in the volume of imaging for each body part, e.g. chest CT. ${ }^{10}$ In another study in the United States, the number of brain imaging declined by as much as $50 \%$ during the pandemic. ${ }^{11}$ 


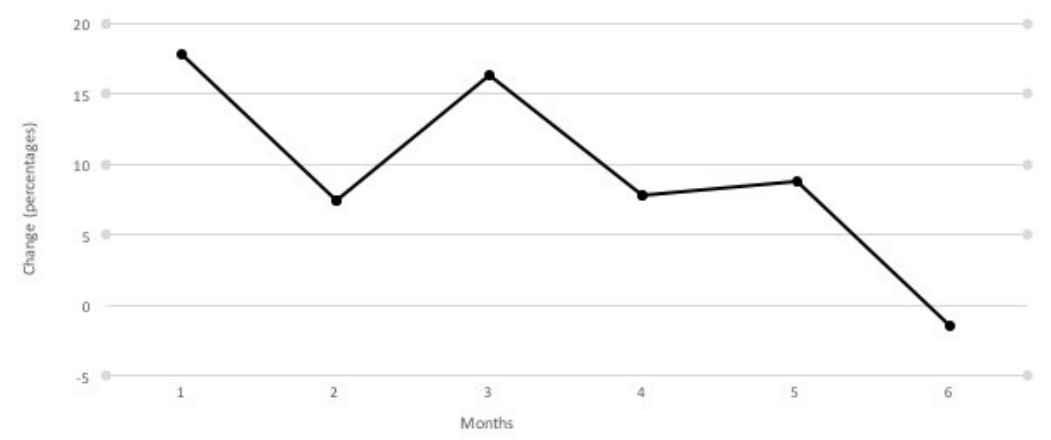

Figure 5. Line Chart to Show the Trend of Income Change Presented as Percentage for All Departments from Month 1 to 6 in the Year 2019-2020 with Respect to Year the 2018-2019.

These changes have substantial economic consequences for the radiology departments. The economic impact has been warned in Germany, Austria, and Switzerland where the nuclear medicine services were notably reduced 3 weeks after the SARS-CoV-2 pandemic. ${ }^{12}$ In the United States, a huge reduction of $50 \%-70 \%$ has been anticipated in imaging volumes for at least 3-4 months. This reduction will be most prominent in outpatient practices. ${ }^{13}$ In the present study, the total income of the radiology departments in 2019-2020 from CT and MRI was only partially offset in the first five Months by the increased total number of chest CTs. The worst economic impact of the pandemic on the radiology departments was observed in Month 6 of 2019-2020. In this Month, although the $1.5 \%$ decrease in the income of these departments may seem insignificant, radiology departments have faced a significant drop in their revenue considering the inflation rate of $35 \%$ in Iran.

The quality of providing service was already compromised when the maintenance service for the imaging equipment had been reduced due to the economic sanctions and Iranian currency (IRR) depreciation, with a 12 -month inflation rate of $34.8 \%$ ending in Month 6 (i.e. Esfand), 2019-2020. ${ }^{14}$ The apparently increased income of the radiology departments from Month 1 to Month 5 (Figure 5) in 2019-2020 compared to the same period in the previous year must be interpreted and adjusted according to the heavy impact of the high inflation rate. This declining trend of income was further accentuated in Month 6 of 2019-2020 when the curve dropped to a negative value. The international community sent humanitarian aids to Iran including diagnostic kits, medicine, and protective equipment while no aids were sent to support the equipment of radiology centers. Needless to say, this negative economic impact may significantly threaten the survival of imaging centers and overutilization may shorten the availability of the CT scanners for the rest of the fight against the pandemic.
The Pros and Cons of Radiology Subspecialization in Iran With the significant increase in the workload of radiologists, the radiology departments in Iran were not fully capable of dealing with the pandemic. A couple of reasons might explain this deficit: first, the number of cardiothoracic imagers available is not sufficient to meet the increased demand in a pandemic of such respiratory diseases in Iran, where there is no dedicated cardiothoracic imaging fellowship program. Second, many radiologists in Iran are working as sonographers without any experience or involvement in cross-sectional imaging. Therefore, health authorities must be prepared for any possible future pandemic by investment and planning for training subspecialized cardiothoracic radiologists as well as other healthcare staff necessary for these situations. This specific shortage of fellowship-trained radiologists in thoracic imaging adds to the already described shortage of medical providers in the country. Although small cities, non-academic centers, and rural and underserved areas may experience the shortages more severely, the need for subspecialty-trained radiologists is felt everywhere. The downside of subspecialization can be highlighted by looking at the odds of chest CT studies to all other CTs in Month 6, 2019-2020 compared to the same odds in Month 6 of 2018-2019 (odds ratio 2-19; Figure 4). This shows how other subspecialties like abdominal, neuro-, or musculoskeletal radiologists may suffer from job insecurity.

Several limitations should be considered when interpreting the results. First, we did not have data from the other 22 provinces of Iran to conduct a comprehensive national survey. However, covering more than $35 \%$ of the urban population could provide important information about the trend and pattern of imaging utilization. Second, three enrolled private centers were excluded from income analysis because of the different tariffs they use for imaging. This exclusion could not significantly skew our results since most of the individuals were referred to the teaching (public) hospitals thanks to the benefits of insurance coverage. Finally, although it was not the 
primary question of this study, data from patients referred to hospitals due to respiratory symptoms could provide more granular data regarding the onset and footprint of COVID-19 in Iran.

In conclusion, the results of this study could provide a valuable overview of the trend and pattern of imaging utilization in a wide multi-province setting during the COVID-19 outbreak in Iran. Compared to the same period in 2018-2019, a significant increase was identified in the acquisition of chest CT studies in Month 3, 20192020 (two months before official announcement of SARSCoV-2 in Iran) without a persistent increase in months 4 and 5. This might be attributed to the seasonal waves of influenza or lack of healthcare system awareness about presence of an emerging viral infection, i.e. COVID-19. This might have caused the underutilization of chest CT in months 4 and 5. The increased burden on the radiology workforce initiated a vicious cycle in which the resulting burnout, financial hardship, and lack of maintenance service worsened the economic repercussions of the pandemic. The shortage of thoracic radiologists and job insecurity for non-thoracic radiologists (including those who strictly focus on ultrasonography) in Iran should also alert the health authorities to choose wiser policies in the future.

Further studies are warranted on a wider scale and a higher temporal resolution of data collection focusing on the imaging and clinical findings to better track COVID-19 footsteps in different countries in more details.

\section{Authors' Contribution}

$A R$ and $A G$ contributed equally as co-first authors. They were involved in the study design, conduct, data gathering, analysis of the data, and writing and editing the manuscript. SAM was involved in statistical analysis, manuscript writing and editing. AK was involved in the statistical analysis. NN, RML, RJR, AD, AH, ASR, MPR, RNM, $\mathrm{HS}, \mathrm{AE}, \mathrm{MA}, \mathrm{GJS}, \mathrm{AS}, \mathrm{MM}, \mathrm{RJ}, \mathrm{MB}, \mathrm{TFL}, \mathrm{MR}, \mathrm{AA}, \mathrm{PD}, \mathrm{HBM}$, and $\mathrm{HH}$ provided data and participated in manuscript writing and editing. MST was involved in study design and conduct, data management and audit, and manuscript editing.

\section{Conflict of Interest Disclosures}

All authors have nothing to disclose.

\section{Ethical Statement}

The study was approved by the ethics committee of the National Institute for Medical Research Development in Iran (ID: IR.NIMAD. REC.1399.011).

\section{Data Sharing Statement}

Data will be available upon request to the corresponding author.

\section{Funding}

None.

\section{References}

1. Dong $E$, Du H, Gardner L. An interactive web-based dashboard to track COVID-19 in real time. Lancet Infect Dis. 2020;20(5):533-534. doi: 10.1016/S1473-3099(20)30120-1.

2. World Health Organization. Coronavirus disease 2019 (COVID-19) Situation Report - 31. 2020 February 20, 2020.

3. Murphy A, Abdi Z, Harirchi I, McKee M, Ahmadnezhad E. Economic sanctions and Iran's capacity to respond to COVID-19. Lancet Public Health. 2020;5(5):e254. doi: 10.1016/S2468-2667(20)30083-9.

4. Kim $\mathrm{H}$, Hong $\mathrm{H}$, Yoon $\mathrm{SH}$. Diagnostic performance of $\mathrm{CT}$ and reverse transcriptase-polymerase chain reaction for coronavirus disease 2019: a meta-analysis. Radiology. 2020;296(3):E145-E155. doi: 10.1148/radiol.20201343.

5. Rubin GD, Ryerson CJ, Haramati LB, Sverzellati N, Kanne $\mathrm{JP}$, Raoof S, et al. The Role of chest imaging in patient management during the COVID-19 pandemic: a multinational consensus statement from the Fleischner Society. Chest. 2020;158(1):106-116. doi: 10.1016/j.chest.2020.04.003.

6. Population and Household of the Country by Province and Sub-province (Shahrestan). Statistical Center of Iran. 2016. Available from: https://www.amar.org.ir/Portals/1/ census/2016/Population-and-Household-by-Province-andShahrestan.xIsx.

7. Li Q, Guan X, Wu P, Wang X, Zhou L, Tong Y, et al. Early Transmission Dynamics in Wuhan, China, of Novel Coronavirus-Infected Pneumonia. N Engl J Med. 2020;382(13):1199-1207. doi: 10.1056/NEJMoa2001316.

8. Ma J. Coronavirus: China's first confirmed Covid-19 case traced back to November 17. 2020 March 13, 2020. Available from: https://www.scmp.com/news/china/society/ article/3074991/coronavirus-chinas-first-confirmed-covid-19case-traced-back.

9. Coletti P, Poletto C, Turbelin C, Blanchon T, Colizza V. Shifting patterns of seasonal influenza epidemics. Sci Rep. 2018; 8(1):12786. doi: 10.1038/s41598-018-30949-x.

10. Naidich JJ, Boltyenkov A, Wang JJ, Chusid J, Hughes D, Sanelli PC. Impact of the COVID-19 Pandemic on Imaging Case Volumes. J Am Coll Radiol. 2020;17(7):865-872. doi: 10.1016/j.jacr.2020.05.004.

11. Tu LH, Sharma R, Malhotra A, Schindler JL, Forman HP. Total and Stroke Related Imaging Utilization Patterns During the COVID-19 Pandemic. medRxiv. 2020. Available from: https:// www.medrxiv.org/content/10.1101/2020.05.20.20078915v1

12. Freudenberg LS, Dittmer U, Herrmann K. Impact of COVID-19 on Nuclear Medicine in Germany, Austria and Switzerland: An International Survey in April 2020. Nuklearmedizin. 2020;59(4):294-299. doi: 10.1055/a-1163-3096.

13. Cavallo JJ, Forman HP. The economic impact of the COVID-19 pandemic on radiology practices. Radiology. 2020; 296(3):E141-4. doi: 10.1148/radiol.20201495.

14. CPI by Provinces in the Month of Esfand of the Year 1398 [Internet]. Statistical Center of Iran. 2020. Available from: https://www.amar.org.ir/Portals/1/CPI\%20BY\%20\%20 PROVINCE\%20\%20in\%20\%20ESFAND\%20\%2098\%20(2). pdf. Accessed May 8, 2020. 